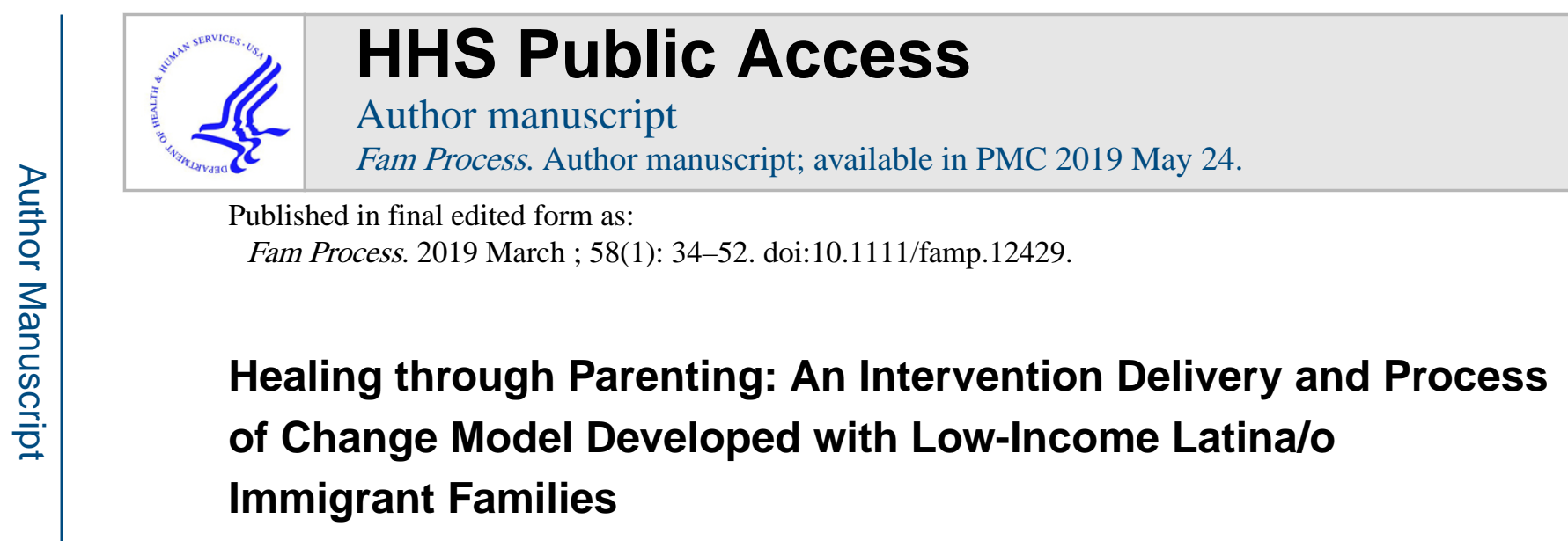

\title{
J. RUBEN PARRA-CARDONA*
}

*Steve Hicks School of Social Work, The University of Texas at Austin, Austin, TX.

\begin{abstract}
Low-income Latinas/os are exposed to widespread mental health disparities in the United States. Most recently, a resurgence of anti-immigration narratives has led vulnerable Latina/o immigrants to experience considerable contextual stressors with multiple deleterious consequences for their overall well-being, including significant disruptions to their parenting practices. Within this context of adversity and despite the multiple benefits associated with parent training (PT) prevention interventions, the availability of contextually and culturally relevant PT interventions remains limited in underserved Latina/o communities. This paper constitutes a contribution to this gap in knowledge by presenting a model of intervention delivery utilized in the dissemination of culturally adapted versions of the evidence-based intervention known as GenerationPMTO. ${ }^{\odot}$ The proposed model also describes a process of change that we have documented in empirical research with low-income Latina/o immigrant parents who have been exposed to the adapted interventions. The manuscript is organized in four sections. First, an overview of the model is discussed, along with a brief summary of major theories. Next, the core components of the model are described, complemented by the presentation of a case study. Finally, implications for prevention and clinical intervention are discussed.
\end{abstract}

\section{Keywords}

Parenting; Parent Training; Cultural Adaptation; Process of Change; Social Justice

Latinas/os ${ }^{1}$ have become the largest ethnic minority population in the United States (US), however, low-income Latinxs continue to be seriously impacted by widespread mental health disparities (SAMHSA, 2015). For example, although meta-analytic data indicate that parent training (PT) interventions constitute the gold standard for preventing child and youth externalizing behaviors, the availability of contextually and culturally relevant PT prevention interventions continues to be scarce in underserved Latinx communities (Gerdes, Kapke, Lawton, Grace, \& Hurtado, 2015).

\footnotetext{
Correspondence concerning this article should be addressed to Ruben Parra-Cardona, Steve Hicks School of Social Work, The University of Texas at Austin, 1925 San Jacinto Blvd. Stop D3500, Austin, TX, 78712. rparra@ austin.utexas.edu.

${ }^{1}$ Although I fully recognize the political importance of the term "Latinx," I utilize the terms "Latinx" and "Latina/o" interchangeably in this document, particularly because our research informs the implementation of parenting initiatives in Latin America. Thus, the use of both terms indicates the current controversy of the term in Latin American countries by scholars and practitioners who caution about the potential risks of imposing a US-based term across Latin American cultures.
} 
Most recently, researchers have documented the ways in which anti-immigration narratives and policies have led to a variety of contextual stressors that negatively impact vulnerable Latina/o populations (Hatzenbuehler et al., 2017; McHugh, 2018), including detrimental effects on their daily parenting practices (Cardoso, Scott, Faulkner, \& Lane, 2018). Thus, there is high need for theoretical models aimed at describing the most effective ways to deliver culturally relevant parenting interventions to underserved Latina/o populations, particularly when considering the multiple contextual and cultural challenges that these populations are currently experiencing throughout the US.

Addressing existing gaps in parenting scholarship as it relates to underserved Latina/o immigrants constitutes an urgent need because effective parenting constitutes a critical protective factor in the lives of children and youth (Michelson, Davenport, Dretzke, Barlow, $\&$ Day, 2013). Similarly, there is a need to better understand the process of change that Latinx parents experience as they are exposed to culturally adapted PT interventions aimed at supporting their parenting efforts.

This paper constitutes a contribution to this gap in knowledge by presenting a model of intervention delivery utilized in the dissemination of culturally adapted versions of the evidence-based intervention known as GenerationPMTO. ${ }^{\odot}$ The proposed model also describes a process of change that we have documented in empirical research with lowincome Latina/o immigrant parents who have been exposed to the adapted interventions. We consider this component of the model to be relevant as nurturing parenting can provide healing experiences for caregivers exposed to intense contextual adversity and adverse childhood backgrounds (Wong, 2009). To clarify, the proposed model was developed based on lessons learned through the implementation of parenting prevention studies with underserved Latinx immigrant families, in an effort to maximize reach with a population that experiences significant challenges to access high-quality mental health services.

\section{HEALING THROUGH PARENTING: AN INTEGRATIVE AND APPLIED MODEL}

Recognizing that the parenting literature is vast and comprehensive and that various parenting models have already been developed and empirically tested (Johnson, Berdahl, Horne, Richter, \& Walters, 2014), the main goal of this paper is to offer a resource to family therapists and other mental health practitioners directly involved in the delivery of parenting interventions to underserved Latinx immigrant populations. Thus, the applicability and relevance of the model for additional diverse populations remains to be demonstrated in empirical research.

The manuscript is organized in four sections. First, an overview of the model is discussed, along with a brief summary of major theories. Next, the core components of the model are described, complemented by the presentation of a case study. Finally, implications for prevention and clinical intervention are discussed.

\section{Core and Informing Theories}

The proposed model has been developed based on lessons from the cultural adaptation of the efficacious parenting intervention known as GenerationPMTO. Specifically, an initial study 
funded by the National Institute of Mental Health (NIMH) had the main objective of empirically testing a culturally adapted version of GenerationPMTO for low-income Latina/o immigrant families with young children, ages 4-11. Most recently, with funding support from the National Institute on Drug Abuse (NIDA), we have adapted the intervention for Latinx immigrant families with young adolescents, 12-14. Although both adapted interventions differ in terms of tailoring of parenting components according to the age of the target child/youth, the core components of both interventions are similar as well as our approach to intervention delivery.

Figure 1 presents a graphic depiction of the proposed model. In the upper left corner, two sets of theories are identified. Core theories constitute main theoretical premises that we consistently use across all parenting sessions. For example, as stated in attachment theory, we address in every group session the need for parents to become a secure emotional base in the lives of their children. In contrast, we rely on two informing theories to maximize the impact of specific intervention components according to the type of life experiences reported by parents. For example, we utilize experiential principles to maximize the emotional impact of role plays and help parents become fully aware of the range of emotions associated with experiencing various instances of discrimination, as well as the ways in which such experiences negatively impact their parenting practices. However, the depth to which we utilize experiential principles varies according to the type of experiences of discrimination reported by parents.

\section{Directionality and Parenting Goals}

The model indicates a sense directionality to illustrate a sequential process of delivery of intervention components, which is associated with a gradual process of change as caregivers are exposed to various parenting practices. The upper section of the model depicts the following key parenting goals that we seek to achieve in a sequential manner: (a) embracing the parenting dream, (b) becoming aware, (c) promoting strengths and nurturing parentchild relationships, (d) teaching children and youth to live within boundaries, and (e) promoting biculturalism.

The ordering of components in the proposed sequence has been determined according to two premises. First, the initial component, embracing the dream, is focused on the need to ground the parenting intervention according to the immigrant experiences that are most salient to parents. Specifically, our cultural adaptation studies have indicated the critical importance of framing the intervention according to the harsh realities of oppression that parents face as immigrants, as well as the motivations they embrace that help them cope with the various forms of adversity that they experience (Parra-Cardona et al., 2016, 2018).

Subsequent components are situated according to the theoretical premises of the GenerationPMTO intervention. This sequence of core parenting components is justified by $40+$ years of empirical research resulting from the implementation of GenerationPMTO with various populations in the US and across the world (Forgatch \& Gewirtz, 2018).

Initiating with the Becoming Aware component, each of the parenting goals is associated with one of the following five core GenerationPMTO components: (a) giving good directions, (b) positive involvement and skills encouragement, (c) limit setting, (d) 
monitoring and supervision, and (e) cultural family problem solving. Finally, the lower section of the figure identifies advocacy and therapeutic experiences that parents have reported in our qualitative empirical studies as particularly salient after participating in the culturally adapted interventions: (a) advocacy for coping with contextual adversity, (b) healing through parenting, and (c) embracing culture.

Because the focus of this paper is centered on describing our intervention delivery approach and the process of change commonly experienced by parents, we provide a brief summary of the cultural adaptation studies of the GenerationPMTO intervention for Latinx immigrant families. We refer the reader to the original reports of the cultural adaptation studies, which include detailed descriptions of adaptations to curricula according to type of target family (i.e., families with young children vs. adolescents), details of the research designs, and quantitative and qualitative findings from the randomized controlled trials (Parra-Cardona et al., 2012, 2016, 2017a, 2018).

An initial version of the model primarily focused on the culturally adapted core components of the GenerationPMTO intervention has been published in the literature (Parra-Cardona et al., 2017b). The current model constitutes a significant expansion of the original model as we have adapted the interventions to more effectively respond to the various contextual and cultural challenges reported by participant Latina/o families. However, because not all families benefit equally from parenting interventions (Leijten et al., 2018), the current model should only be used as an informative case study describing a prevention approach implemented with low-income Latinx immigrants.

\section{Core Theories}

Liberation psychology-Grounding parenting interventions in social justice principles. We are experiencing an unprecedented time in the US characterized by a resurgence of nationalist narratives that are particularly deleterious to vulnerable and underserved diverse populations. Among affected groups, low-income Latina/o immigrants have become the target of various expressions of anti-immigration narratives and policies, which obscure the essential contributions that this population offers to the US society and economy. The current anti-immigration climate also deviates attention from widespread human rights violations that underserved Latinx immigrants are experiencing in the country, such as labor exploitation, extraordinary barriers to access basic health-care services, discrimination, and the traumatic impacts of forced family separation (Cardoso et al., 2018; McHugh, 2018).

Researchers have documented how these contextual challenges are disrupting parenting practices in Latina/o families in critical ways, leading to serious child and adolescent adjustment problems (Cardoso et al., 2018). For example, studies indicate that Latinx adolescents' perceptions of discrimination experienced by their parents are associated with increased risk for youth internalizing problems and lower self-esteem (Park, Du, Wang, Williams, \& Alegria, 2018). We have recently published qualitative reports of Latina/o youth experiencing suicidal ideation stemming from bullying perpetrated by peers who mock youth due to the undocumented immigration status of their parents (Parra-Cardona et al., 2018). Based on this context of oppression, exhortations by scholars indicating the need to 
overtly address issues of discrimination in prevention and parenting interventions have become urgently pressing (Cardoso et al., 2018; Unger, 2015).

In response, we have embraced liberation psychology as a guiding theory for our work with underserved Latina/o communities (Martín Baró, 1977). Its developer, Martín Baró, vehemently argued that psychology and other mental health sciences must always be evaluated according to their relevance within specific historical and socio-political contexts. Without such a critical analysis, mental health intervention and research can become "a refined instrument to maintain the status quo," rather than a liberating experience for populations exposed to structural and institutional oppression.

Liberation psychology can be considered a social justice theory as it has at its core the analysis of oppressive structures in society. In contrast to multicultural theories which do not uniformly address issues of power and privilege associated with various expressions of diversity, liberation psychology has as a core premise that individuals in society will be subject to current structures of power and privilege according to the diversity they represent (Martín Baró, 1977). For example, people of color, non-European immigrants, and women in the US continue to be exposed to dynamics of oppression associated with racism, nationalism, and patriarchy.

Liberation psychology is not a clinical theory per se, therefore, it is up to interventionists to translate the theory into prevention and clinical practice. For example, we have actively pursued funding for the past decade to ensure that our parenting interventions are offered free of charge in low-income communities. Furthermore, our culturally adapted parenting prevention interventions explicitly address issues of discrimination in intervention curricula, including ways in which immigrant parents can monitor how these stressors impact their daily parenting practices. We also offer advocacy services to every family participating in our parenting programs to help them obtain resources they need to cope with various contextual challenges, ranging from access to health and mental health services to immigration legal services (Parra-Cardona et al. 2018).

Critical race theory-Teaching children and youth to live in a racially stratified society. Critical race theory refers to a body of literature converging on the principle that "race is a central component of social organizations and systems, including families" (Burton, BonillaSilva, Ray, Buckelew, \& Freeman, 2010, p. 442). Additional premises state that racism is institutionalized and all individuals within racialized social systems can contribute toward the reproduction of these systems. Within critical race theories, racial socialization refers to the study of "overt and covert behaviors parents use, over and above those responsibilities shared by all parents, to psychologically prepare children for success in a racially stratified American society" (Peters, 1985, p. 562).

Incorporating critical race theory premises in the proposed model has helped us to address in the parenting curricula issues of race, racism, and related coping strategies. For example, in the initial parenting sessions, we facilitate group conversations focused on how families have been impacted by racial and immigration-related discrimination, as well as the coping strategies they utilize to cope with this adversity. In addition, we invite parents to reflect 
about the ways in which effective parenting constitutes a salient experience for ethnic minority children to be more resourceful in a racially stratified society.

Social learning, coercive theory, and cultural adaptation-Promoting effective and nurturing parenting. GenerationPMTO constitutes the cornerstone of our applied program of prevention parenting research. This intervention is based on a model of social interaction learning theory (SIL; Forgatch, Patterson, DeGarmo, \& Beldavs, 2009). In essence, the SIL model specifies that effective parenting practices and the quality of interactions parents have with their children constitute the most proximal mechanism for promoting successful child adjustment. The GenerationPMTO intervention is also informed by coercion theory, which was originally developed by Gerald Patterson and colleagues (Forgatch et al., 2009). Briefly, coercive theory assists clinicians by analyzing the ways in which aversive behaviors by children and youth can gradually increase in intensity and become recurrent if parents do not practice effective and nonpunitive parenting practices.

A major premise of the GenerationPMTO intervention refers to its theoretical mediational model. Thus, the intervention is delivered primarily to parents as it is expected that by enhancing their parenting practices, the resulting effect will be a successful child and youth development and sustained reductions of children and youth internalizing and externalizing behaviors (Forgatch et al., 2009). Studies consisting of the integration of findings from multiple parenting trials, also known as meta-analyses, indicate that parenting interventions informed by PT principles such as the core components of the GenerationPMTO intervention constitute one of the most effective alternatives to promote prosocial behavior and reduce coercive behaviors (Michelson et al., 2013).

Cultural adaptation of GenerationPMTO-Cultural adaptation refers to the process by which evidence-based interventions originally developed with minimal representation of diverse populations can be effectively adapted according to the most relevant cultural values and traditions of ethnic minority families (Barrera, Castro, Strycker, \& Toobert, 2013).

Domenech Rodríguez et al. (2011) conducted the first adaptation of GenerationPMTO for Latino/a immigrant populations and the resulting adapted intervention was titled "CAPAS: Criando con Amor, Promoviendo Armonía y Superación” (Raising Children with Love, Promoting Harmony and Self-Improvement). Following this initial adaptation, our research group examined the impact of differential cultural adaptation. Specifically, we implemented a study in which we compared the original version of the CAPAS intervention against a CAPAS-Enhanced intervention in which parenting components were complemented by sessions focused on immigration-related challenges, discrimination, and biculturalism. Participant families in this study were low-income first-generation Hispanic families with one target child ages 4-12 (Parra-Cardona et al., 2017a).

Intervention effects indicated that both adapted interventions significantly improved the quality of all parenting practices when compared to the control condition at 6-month followup. However, when compared to CAPAS and control conditions, statistically significant higher improvements on child internalizing and externalizing behaviors were associated with CAPAS-Enhanced. Qualitative findings indicated that the incremental effects associated 
with CAPAS-Enhanced were associated with parents being able to adapt their parenting expectations and practices according to specific contextual challenges such as discrimination and within-family cultural conflicts (Parra-Cardona et al., 2018). These findings are highly relevant as they indicate the importance of overtly addressing issues of contextual adversity and discrimination in parenting prevention interventions (Cardoso et al., 2018; Unger, 2015).

Most recently, we empirically tested the impact of the CAPAS-Enhanced intervention with Latina/o families with adolescents, ages 12-14 (Parra-Cardona et al., 2018). In concordance with our study findings with families with young children, CAPAS-Enhanced had a positive impact on parenting and youth outcomes. Furthermore, qualitative data indicated that parents particularly valued the overt focus of the intervention aimed at helping them address issues of discrimination and parent-adolescent cultural conflicts.

Attachment theory-The power of having a secure base in life. Attachment theory highlights the importance of establishing secure and predictable relationships between caregivers and children and youth (Cassidy, 1999). An extensive body of literature describes the ways in which the parent-child emotional bond is particularly relevant for human development, with the so-called insecure attachment styles indicating challenges to establish such bonds. In contrast, a secure attachment indicates a parent-child relationship characterized by effective emotional connection and stability. In such a relationship, children and youth have the certainty that parents will always be a source of comfort, protection, and support for them (Cassidy, 1999).

The parenting interventions we offer are preventative in nature and are delivered in a group format to maximize reach. The parenting program for families with youth consists of nine manualized sessions and the adapted intervention for families with young children consists of 11 sessions. Because our focus is preventative in nature, we offer a limited number of individual sessions if parents report intense childhood backgrounds of child maltreatment and neglect or considerable contextual adversity. The individual sessions have the main purpose of providing parents the opportunity to process in more depth issues addressed in the parenting program and assess whether they need more specialized services. If that is the case, they are referred to services to one of our community collaborators.

Based on empirical findings from our studies, we have corroborated the positive impact of overtly addressing core attachment principles with parents (Parra-Cardona et al., 2016, 2018). However, rather than seeking to address in depth caregivers' issues of insecure attachments with their own parents, our approach has focused on conveying to parents the importance of being the sources of attachment security for their children, as well as the ways in which specific GenerationPMTO parenting skills can promote attachment security (e.g., consistently praising children for good behavior).

Emphasizing the importance of promoting a secure attachment with children through daily parenting practices is particularly important if parents experience reactivity when exposed to specific intervention components. For example, parents who have experienced emotional neglect as children can react negatively when presented with components emphasizing the need to praise children for good behaviors. Paradoxically, as parents strengthen a secure 
emotional relationship with their children by engaging in nurturing and effective parenting skills, they gradually see in a different light the relationship with their own parents. In fact, it is common for parents to express that their own experiences of success as parents constitute a precursor for understanding their parents' deficient caregiving behaviors. Parents also frequently report how their own parenting experiences facilitate a process of forgiveness for the maltreatment or neglect that they experienced as children (Parra-Cardona et al., 2016).

\section{Informing Theories}

Experiential family therapy-According to one of the core premises of experiential family therapy, a process of change is facilitated when individuals can embrace a wide range of emotions associated with specific life experiences and interpersonal relationships, particularly if such feelings have been suppressed over time (Satir, Banmen, Gerber, \& Gomori, 1991). However, rather than engaging parents in extensive clinical experiential interventions, we have adopted core premises of experiential theory to maximize the impact of specific PT components.

For example, role plays are at the cornerstone of the GenerationPMTO intervention as they are used to help parents experience effective and ineffective parenting skills from the perspectives of both the parent and the child (Forgatch, Bullock, \& Patterson, 2004). Informed by experiential theory principles, we adapt parenting role plays according to daily immigration stressors reported by parents. To illustrate, prior to engaging parents in limitsetting role plays, we invite caregivers to become aware of the ways in which cumulative stress has negatively impacted their discipline practices (e.g., increased yelling at children due to accumulated stress).

Depending on the type and intensity of stressors reported by parents, we engage parents in brief experiential exercises to help them identify the layers of emotional distress associated with specific immigration-related challenges. For instance, when reflecting on daily experiences of stress when leaving home, parents have created sculptures of people pointing fingers at them in a blaming posture. This brief exercise allows parents to reflect on the emotional impact of narratives to which they have been exposed in various settings reflecting anti-immigration sentiments. Once parents access and verbalize the underlying emotions, we engage them in emotion-regulation exercises and invite them to focus on the ways in which their parenting dreams can act as buffers against these expressions of oppression. Finally, we proceed to deliver the PT components focused on limit setting.

Contextual family therapy-Relational and transgenerational justice. Key premises of contextual family therapy highlight the importance of achieving fairness in the parent-child relationship, the need to identify detrimental transgenerational legacies, as well as the importance of promoting parental accountability as a key precursor of new legacies (Boszormenyi-Nagy \& Krasner, 1986).

Due to the brief nature of our parenting interventions, we have prioritized addressing salient components of contextual therapy. First, we invite parents to identify the parenting legacies they received, which, based on the population we serve, oftentimes consist of childhood experiences of child maltreatment and neglect. In addition, contextual family therapy 
highlights the need for parents to embrace parental accountability for the caregiving of their children. Thus, we address with caregivers the importance of conceptualizing parenting as a relational experience in which parents should be the primary sources of comfort, protection, and emotional nurturance for children. That is, the notion of asymmetry in which the "give and take" process relies primary on the caregivers' unidirectional contributions to the relationship, without expecting children to reciprocate at an equal level which would constitute symmetry, a characteristic of adult romantic relationships.

The positive impact of informing GenerationPMTO according to contextual therapy premises has been consistently reported by parents in our qualitative evaluations as caregivers have reported "becoming aware" of the impacts associated with specific parenting legacies in their families of origin, such as narratives indicating that expressing love is detrimental for Latinx children who live in a society where they will most likely experience discrimination (Parra-Cardona et al., 2016, 2018). Thus, focusing on key contextual theory premises has allowed us to help parents see themselves as "gatekeepers" of detrimental transgenerational parenting legacies, as well as carriers of new legacies characterized by predictability, unconditional support, and continuous expression of love.

It is critical to highlight that we emphasize to parents the ways in which destructive legacies constitute expressions of the contextual adversity and oppression experienced by families. For example, the vast majority of parents in our studies were raised in impoverished communities with caregivers exposed to intense poverty, shortage of basic services, including lack of access to mental health services, and parenting prevention programs. Similarly, parents in our studies have consistently reported exposure to various instances of discrimination in the US as low-income immigrants (Parra-Cardona et al., 2016, 2018).

Thus, it is important to invite parents to reflect about the benefits of nurturing parenting practices that they were not likely to experience as children, additional intervention components such as the ones focused on racial socialization, play a key role to address parents' concerns about future instances of discrimination that their children are likely to experience in the US.

\section{PROCESS OF CHANGE: ENHANCING PARENTING PRACTICES}

\section{Embracing the Parenting Dream}

Efficacious parent training interventions are characterized by intervention delivery approaches focused on strengths-based perspective (Michelson et al., 2013). However, parenting interventions that emphasize parenting practices consisting of praise and emotional nurturance can represent a challenge for parents with backgrounds of continuous exposure to contextual adversity (Johnson et al., 2014). With regard to the populations we serve, we have consistently documented in our studies the high likelihood for parents to have experienced intense challenges in their countries of origin, persistent exposure to poverty, community violence, food insecurity, as well as childhood backgrounds of maltreatment or neglect (Parra-Cardona et al., 2016, 2018). Thus, a key objective in the initial group session consists of promoting a strong sense of motivation for parents to remain engaged in the intervention. This is an essential goal to accomplish because our parenting programs are not 
mandatory in nature. Parents have consistently reported across our parenting trials that experiencing this initial sense of motivation was a critical precursor for them to remain engaged in the intervention (Parra-Cardona et al., 2018).

Thus, we conduct a guided imagery exercise in the initial session in which they visualize various phases of their children's development, including life challenges that they are likely to encounter (e.g., being offered drugs for the first time). Next, we engage parents in a debriefing conversation by focusing on their parenting motivations and aspirations. Parents have confirmed in our qualitative evaluations that this technique constitutes a meaningful experience for them because they can verbalize and process with other caregivers their parenting dreams and hopes (Parra-Cardona et al., 2016, 2018). These findings correspond with empirical research indicating that parenting desires and aspirations constitute a key predictor for engagement in parenting interventions (Wong, 2009).

Parenting within contexts of contextual adversity and oppression-In the initial sessions, we promote group conversations focused on strategies to pursue their parenting dreams within the context of immigration in the US. This contextualization is critical, particularly as it refers to addressing the ways in which chronic adversity and oppression can gradually erode the participants' motivation to enhance their parenting practices.

We rely on role plays to elicit these conversations. For example, we demonstrate through role plays how ineffective parenting practices (e.g., yelling back at children) are greatly influenced by cumulative immigration-related stress. Common stressors reported by parents include labor exploitation, fear of immigration authorities, lack of access to basic health care services, and despair resulting from prolonged family separation.

In the initial session, we also introduce parents to the advocacy component of the intervention, which consists of conducting a needs assessment with each family and designing a plan to help them address a variety of challenges, ranging from facilitating access to immigration legal services to enrollment in welfare assistance programs. We consider the advocacy component to be a clear application of liberation psychology principles, particularly because if we were to only promote conversations with parents about the adversity they experience without offering specific alternatives to address it, we could perpetuate structural dynamics of oppression.

Finally, the initial component of the model includes conversations focused on participants sharing strategies aimed at helping their children cope with racial discrimination. This component is informed according to racial socialization principles as applied to Latina/o immigrant populations. For example, parents have reported strategies they use to emphasize to their children the need to disregard racist comments by peers and remain proud of their Latina/o heritage. We complement this process by encouraging parents to brainstorm in group alternatives to increase their social support networks, including new connections with other participants of the parenting program. 


\section{Becoming Aware}

Having addressed the aforementioned initial intervention objectives, we transit to deliver the core components of the GenerationPMTO intervention.

Giving good directions-According to GenerationPMTO principles, self-awareness is promoted by helping parents identify the strengths and limitations of their parenting practices (Forgatch et al., 2004). The model specifies the use of role plays to achieve this objective. Specifically, parents are first exposed to role plays to help them distinguish "bad versus good directions." Role plays demonstrating bad directions illustrate to parents ineffective parenting commands such as vague instructions, yelling, or threats. Next, parents are engaged in good directions role plays, consisting of succinct, specific, and emotionregulated parental commands. Humor is essential when engaging parents in these role plays as they immediately connect with ineffective directions that they have used in the past. Therefore, it is critical to normalize with participants that giving deficient directions is a struggle experienced by all parents. Participants have consistently highlighted the positive impact of this component by emphasizing how good directions role plays helped them "become aware" of specific limitations of their parenting practices.

\section{Promoting Strengths and Nurturing Parent-Child Relationships}

Positive involvement and skills encouragement are two core components of the GenerationPMTO intervention that have the goal of promoting nurturing parent-child relationships, as well as strengths in children and youth (Forgatch et al., 2009). Briefly, positive involvement refers to promoting nurturing and loving ways of interacting with children and youth. Skills encouragement refers to utilizing tools such as reinforcement of positive behaviors that will help children and youth become self-sufficient.

As we address these components, parents tend to react in distinct ways. The majority express an interest in learning new parenting skills and are open to test them at home. In contrast, some parents remain reluctant to the idea that children should be incentivized and rewarded for good behaviors. Whenever parents react with frustration or skepticism when presented with these tools, we provide a strengths-based reframe such as, "We thank you for honesty. This demonstrates that you are committed to being the best gatekeeper in the lives of your children. For now, we just ask you to keep coming to group and listen to the experiences reported by other group members who will start using this technique at home. In the end, you will decide what is the best for your children, because as parents, you are the leaders of your families and you know what is best for them."

Such a nondefensive stance is fruitful as many parents who are initially reluctant to test the new parenting practices, gradually engage in a process of reflection about their own childhood experiences by listening to testimonies of success shared by group members. Others slowly reflect on parenting assumptions that have informed their parenting practices and that have consisted of an emphasis on discipline with limited recognition of children's good behaviors. 
As we debrief the participants' experiences who try the new techniques at home, we are particularly attentive to parents who report that these skills connect them with difficult memories such as past exposure to maltreatment or neglect as children. As caregivers share their testimonies, we validate the distress and sense of loss associated with these disclosures. Processing these experiences and validating the resulting range of feelings constitute a core tenet of experiential family therapy theory. Once we validate the participants' hurtful memories, we encourage parents to recognize the control they have with regard to the type of relationships they want to establish with their children. Sensitivity to these issues is important, as highlighted by one mother who was exposed to punitive parenting experiences as a child: "[T]his process is very hard ... when you try these parenting practices, you see everything that you never received from your parents."

Further, the GenerationPMTO model clearly indicates that parents must experience hope and an increased sense of parenting competence at the completion of every session. Therefore, informed by contextual family therapy principles, we highlight to parents the power they have to offer a new legacy to their children consisting of effective, safe, and nurturing parenting practices. As parents test the parenting skills at home and experience success with their children, a "snowball effect" takes place in group sessions. This process is critical for parents who were initially reluctant to test the skills, as they witness the sense of satisfaction and joy from parents reporting improved children's behaviors and higher quality of parentchild relationships.

\section{Teaching Children and Youth to Live within Boundaries}

Empirical research shows that limit-setting parent trainings components are well received by Latino/a immigrant parents due to Latino/a cultural values that emphasize the importance of promoting respect in interpersonal relationships (i.e., respeto), as well as family cohesion and harmony (i.e., familismo; Stein \& Guzman, 2015).

GenerationPMTO exposes parents to limit-setting practices consisting of mild and nonpunitive consequences for misbehavior, such as time outs for young children and brief removal of privileges for children and youth. There is also a focus on strengthening skills that are necessary to master limit-setting practices, such as the ability to regulate emotions while managing conflictual interactions with children and youth. As described in the introduction section, we rely on experiential theory to inform limit-setting role plays and help parents identify the ways in which immigration-related stressors negatively impact their limit-setting practices.

Monitoring and supervision-Our studies indicate that although parents are enthusiastic about this component, they usually need to expand their conceptualization of risk. For example, when discussing risks of sexual abuse, it is common for parents to express surprise by learning that the majority of assailants are family members or close acquaintances to survivors. We also focus on supervision issues related to technology as even if parents report living at poverty levels, electronics are easily accessible by families and youth. Based on feedback from parents, we are expanding the curriculum for families with adolescents to address in more depth issues of adolescent sexuality and supervision. We 
refer the reader to the original research report for a detailed description of these research findings (Parra-Cardona et al., 2018).

\section{Embracing Culture}

Biculturalism refers to the ability of families to integrate elements from two cultures while respecting individual cultural differences. Family intervention studies indicate that promoting biculturalism has a positive effect if parents and children experience conflictual interactions due to individual cultural differences (Stein \& Guzman, 2015). For instance, it is common for immigrant youth to be more oriented toward US cultural values and traditions, including a preference for English language. This oftentimes constitutes a considerable challenge for foreign-born parents who expect their children to primarily speak Spanish and embrace traditional Latino/a cultural values.

In a brief format, we have adapted techniques previously reported in the literature as useful for promoting biculturalism in immigrant families (Stein \& Guzman, 2015). First, we foster conversations with parents to help them identify their own cultural identities and preferences. Next, we coach parents through role plays to have similar conversations with their children at home, with the primary goal of learning about their children's cultural preferences. We emphasize to parents the importance of validating their children's feedback without expressing judgments or criticisms, particularly when it refers to adolescents. As parents report back to their group on the conversations they had with their children, many caregivers express feeling surprised about learning about their children's cultural preferences, as well as sadness or anger if their children's preferences are not oriented toward Latino/a cultural values or traditions.

After validating the parents' wide range of experience, we engage with them in role plays aimed at validating their children's cultural preferences and communicating from a strengths-based perspective, their cultural hopes for them. For example, rather than having a conversation in which parents mandate adolescents to speak Spanish, we coach parents on communicating to their children the multiple benefits associated with being bilingual. The qualitative evaluations we have conducted of this component indicate high participant satisfaction with this approach (Parra-Cardona et al., 2018).

Cultural family problem solving. As we implemented our initial prevention studies, parents spontaneously adapted the family problem solving component to help them promote biculturalism in the family. We were thrilled about this process as it highlights the strong sense of resilience and creativity of immigrant families. Briefly, the original GenerationPMTO component consists of addressing family challenges by engaging all family members in a cooperative solution-seeking process. Thus, parents are provided with tools focused on engaging all family members in a brainstorming process aimed at evaluating potential alternatives, finalizing with the selection of a preferred solution that is acceptable to all family members.

We encourage families to adapt this component according to their own resources and expectations related to family biculturalism. For example, parents reported that planning trips to their countries of origin constitutes an ideal alternative to help children and youth 
connect with the privileges they experience in the US, while also placing children in a context that makes them experience humility by becoming aware of their limitations (e.g., recognizing language limitations).

\section{CASE STUDY: CHANGING LEGACIES AND OVERCOMING THE ODDS}

\section{Background}

Angelica and Eduardo are Latino/a immigrants and have resided in the US for over a decade.

2 Both parents reported ages in the mid-40s at the time of enrollment in the parenting prevention program. Their primary motivation to relocate to the US was their desire to achieve financial stability and security for their family, as they lived in a rural Mexican community characterized by extreme poverty and widespread violence associated with the drug cartels. At the time of their participation in the parenting program, Angelica had initiated an immigration petition to become a permanent resident.

They enrolled in the parenting program based on concerns for their US-born adolescent child Julia, who was 13 years of age at the time of enrollment. Julia was showing increasing defying attitudes such as talking back to parents and not complying with requests by her mother. Eduardo was not residing at home at the time of intervention delivery as he secured a temporary job out of the state. However, they decided for the family not to relocate as Eduardo's job was temporary. To ensure that Angelica and Eduardo adopted a common approach to parenting, we consistently checked with Angelica about Eduardo's reactions to the parenting skills she gradually implemented at home as a result of participating in the program. Eduardo also attended one review session during one of his home visits. Eduardo was very open and supportive of the intervention content and approach.

\section{Engaging in the Intervention}

Angelica reported initial hesitancy about enrolling in the parenting program and decided to attend the initial sessions to make a determination about continuing to attend the intervention. Eduardo expressed to Julia that he would support her participation from the distance.

To Angelica's surprise, the guided imagery exercise in the initial session was a key motivator to keep her attending the program as she reflected on the protective factors associated with effective parenting practices. The initial sessions were also important for Angelica as she listened to testimonies by other parents who reported experiences of adversity due to their ethnicity and immigration status. Prior to participating in the parenting program, Angelica had difficulties finding friends with whom she could openly share the challenges she had experienced as an immigrant, particularly because she struggled with identifying trustworthy people who would validate her experiences. As she expressed, "it is hard to realize that we only want to offer a good life to our family and we are committed to working hard but many people in this country do not like us."

\footnotetext{
${ }^{2}$ Participants' personal details and characteristics have been modified to protect the anonymity of all family members.
} 
Angelica also connected with struggles shared by parents as they discussed cultural conflicts with their children. In fact, she reported being particularly impacted by a role play in which the interventionists showed a parenting situation consisting of a conflictual interaction because the son refused to speak Spanish at home. Angelica also reported that the home practice assignment for that session was difficult to accomplish because it consisted of inviting Julia to reflect about characteristics of the US and Latino/a cultures that she liked.

In group, Angelica reported that this home practice assignment was challenging because Julia expressed not seeing a value in speaking Spanish and preferred US celebrations such as Thanksgiving. Although Angelica felt reactive toward Julia's feedback, she remembered that a primary task of this assignment was to report back to the group her experiences and avoid any confrontation with Julia. Back in group, Angelica wondered if she was overreacting or was a "deficient or selfish" parent. However, as she started listening to similar experiences shared by other parents, Angelica felt relieved and free to share openly in group her reactivity and disappointment in response to Julia's feedback. As she expressed, it was liberating "poder sacarlo de mi pecho" (getting it out of my chest).

As Angelica processed with other parents her struggles related to cultural conflicts, she learned effective strategies to deal with this issue, including the fact that cultural differences, rather than Julia herself, was the problem that had to be addressed. Angelica reports that the strategies focused on promoting biculturalism were particularly helpful as she gradually developed ways to express her cultural hopes to Julia but in caring ways. For example, Angelica shared with her daughter experiences of discrimination that she lived in previous workplaces, as well as the fact that she could not aspire to seek better quality jobs with decreased risk for discrimination because she was not bilingual. These disclosures prompted unexpected reactions from Julia. For example, one day Julia expressed to Angelica that it was sad for her to learn about all the challenges Angelica and Eduardo have endured as immigrants and that she felt grateful to know that she could go to school and have an education in the US.

\section{Embracing Healing and Change}

Angelica identified two additional parenting components that had a significant impact on her. The first referred to promoting positive involvement by giving good directions. Specifically, Angelica refers that although she laughed when the interventionists engaged in humorous role plays showing deficient ways of giving commands to adolescents, she became aware that conflictual interactions with Julia started with inefficient commands given by her. It was precisely while debriefing these role plays that Angelica recognized that she was mainly blaming Julia for her lack of compliance, without assuming personal accountability for the role she had in such conflictual interactions. As she expressed, "me dí cuenta que yo era la del problema, no mi hija" (I realized I was the problem, rather than my daughter).

Angelica also reflected about the session on encouragement and the need to consistently motivate prosocial behavior in youth by focusing on praise and recognition of adolescents' good behaviors. This was a particularly difficult intervention component for Angelica to assimilate as it represented a clear contrast with the parenting experiences she lived as a 
child, which included maltreatment by her father. Specifically, Angelica reported always aspiring to attend college but her father was never kind to this idea. In fact, when she transitioned to middle school, he told her that he was not responsible for providing her with school supplies as she had already completed her elementary education. Angelica remembered this experience with great pain and is thankful for her mother's support, who, although she did not have economic power in the couple relationship, always found ways to gather the resources Angelica needed to complete high school.

As she reflected on these experiences in group, she found herself sharing common childhood experiences with other parents. For instance, she reflected about intense challenges that her family experienced in her country of origin, such as poverty, lack of medical services, and community violence. Angelica also reflected about the harsh realities for men in rural Mexico, as it is common for them to be exposed to strenuous working conditions and minimal wages with no benefits. Based on these group conversations, she disclosed an increased understanding of the oppression that her own father experienced in Mexico, as well as the ways in which such experiences of oppression prevented him from being an emotionally nurturing father.

Thus, Angelica expressed the need to "cambiar la herencia familiar para Julia" (to change the family legacy for Julia), with the understanding that she had alternatives that her parents, and particularly her father, did not have such as being able to raise her child in emotionally nurturing ways, without having to heavily rely on harsh parenting practices. As she engaged in testing the positive involvement and skills encouragement parenting skills, she was surprised by seeing Julia's compliance with tasks assigned to her, which was characterized by gradual behavioral changes and consistent praise for good behaviors. According to Angelica, "fué como si mi hija cambiara de la noche a la manana!" (It was like my daughter changed overnight).

Angelica considered that the limit setting and supervision components were significantly easier to integrate as they strongly aligned with the goals she wanted to pursue when she first enrolled in the parenting program. The monitoring and supervision component also closely aligned with supervision practices Angelica was already implementing. Thus, she reported enhancing practices such as monitoring the use of electronics, as well as supervision skills aimed at preventing early age drug use initiation.

\section{Biculturalism: Living Between Worlds}

Angelica reported that the most relevant lesson she learned after exposure to the biculturalism component referred to understanding the negative impact associated with demanding from her daughter to embrace specific cultural preferences. Instead, Angelica reflected on the need to communicate to Julia that she had the freedom to make these decisions, while also inviting her to reflect on the motivation informing her parents' encouragement related to cultural issues. For example, Angelica reported that one of the most fruitful conversations with Julia consisted of sharing with her the benefits of becoming bilingual as a way to overcome adversity and pursuing a professional path with reduced risk of exposure to work exploitation and experiences of discrimination. 


\section{Advocacy: Beyond Parenting Practices}

Angelica and Eduardo did not request advocacy services while they were attending the parenting group. However, a couple of months after completing the parenting program, Angelica was notified by her immigration attorney that she was subject to deportation due to issues of her immigration background. The news had a profound impact on the family but was particularly deleterious for Julia, as she became increasingly anxious about Angelica being deported. Within days of the notification, Julia's grades dropped substantially and she became notoriously isolated and distraught.

As days passed on, Julia refused to attend school and expressed her desire to stay home with Angelica. At this juncture, our project manager ${ }^{3}$ received a call from Angelica describing the situation and requesting our support by providing advocacy support for her immigration petition. Thus, the project manager interviewed Angelica to complete a detailed timeline of the immigration notification and the associated emergence of Julia's symptomatology. Having integrated an initial draft, the first author worked closely with the project manager on a petition that provided detailed background of the family, as well as a careful description of the considerable improvements observed in Angelica's parenting practices.

The document also detailed the behavioral changes observed in Julia and the intense anxiety she was experiencing as a result of the immigration notification. Great emphasis was given to highlight the emotional hardship that Julia would experience if Angelica was deported. A closing argument focused on providing details of Angelica's progress throughout the parenting intervention, clearly demonstrating that Angelica was the only parent with full knowledge of an evidence-parenting intervention that would be critical to ensure Julia's mental health in the present and future.

After Angelica's immigration attorney submitted the response to immigration authorities, we kept regular contact with Angelica and referred her and Julia to a local therapist to help them cope with the intense anxiety they were experiencing. Fortunately, the immigration review was favorable, the deportation proceeding was stopped, and Julia was granted immigration documented status. The news dramatically improved everyone's emotional well-being, including Julia's symptoms which drastically decreased. Angelica expressed that as she experienced certainty for the future, the skills learned in group became a daily reminder of "las oportunidades que tengo cada día de demostrarles a mis hijos mi amor por ellos" (the opportunity I have everyday to show my love for my children).

\section{IMPLICATIONS FOR PREVENTION AND CLINICAL INTERVENTION}

Informed by the premises of the proposed model, we offer specific recommendations for prevention intervention, as well as suggestions for potential clinical applications.

\footnotetext{
${ }^{3}$ We express our deep gratitude to Gabriela López Zerón, Ph.D., who was the project manager for this study and had a key role in preparing the response letter to immigration authorities. Her role in this case was invaluable.
} 


\section{Parenting Prevention Interventions}

Parenting prevention interventions constitute a highly relevant area of practice for family therapists, particularly because of the high applicability of family therapy theories for enhancing prevention programs. Furthermore, prevention interventions can increase the level of reach to underserved communities that experience significant barriers to access high quality mental health services.

Despite the multiple benefits associated with parenting prevention interventions, delivery approaches based on group-based formats have limitations that must be recognized. For example, enrolled families must have at least one target child/adolescent exhibiting mild- to moderate-problem behaviors. Otherwise, the limited number of sessions that characterize manualized prevention interventions will not be sufficient to adequately respond to the needs of families whose children exhibit symptomatology in clinical ranges. To address the lack of eligibility, we rely on a referral network of providers of mental health services with a history of serving low-income Latinx communities. In addition, because we also screen for domestic violence (DV), we make necessary referrals to a local DV program that specializes in serving Latina/a populations.

Participants in parenting interventions may also disclose sensitive information as they are exposed to various topics in the parenting curriculum. Therefore, interventionists must be able to identify chronic emotional distress among parents, as well as unaddressed mental health problems. Thus, brief individual sessions between group meetings can be offered to parents to determine the need for referrals to specialized therapeutic services. If it is determined that parents are not in clinical ranges, we have learned over the course of implementing the parenting programs for several years that the intervention itself can facilitate a healing process for parents as they engage in nurturing parenting practices with their own children. This healing process has been consistently expressed by parents in the qualitative evaluations as caregivers can understand the childrearing challenges experienced by their own parents, which allows them to give a new meaning to the experiences of maltreatment and neglect that they experienced as children (Parra-Cardona et al., 2016, 2018).

\section{Clinical Intervention}

Although the current model has been developed based on lessons learned from implementing prevention parenting interventions, family therapists may find specific premises of the model to be relevant for clinical practice with Latinxs and other diverse populations. In fact, cross-cultural parenting research indicates that core premises and components of efficacious parenting interventions can be perceived as similarly relevant across cultures and ethnic groups (Bornstein, 2012).

For example, our research with Latinx populations in the US and Mexico, as well as additional GenerationPMTO studies in the US and Europe, indicates the need to maintain fidelity to the core components of PT interventions. A key dimension of fidelity refers to following a clear sequence of delivery of parenting components. For example, the sequence described in the model is grounded in empirical evidence indicating that prior to engaging in 
enhancing limit-setting practices, it is critical to reinforce the parent-child emotional bond. Thus, we recommend clinicians to become thoroughly familiar with the premises of selected PT interventions and follow recommended guidelines for intervention delivery. We refer the reader to the original GenerationPMTO website (https://generationpmto.org), which offers a wide variety of resources for clinical practice, including free access to relevant and illustrative clinical publications.

\section{The Healing Power of Culture}

It is of critical importance for family therapists to remain attentive to the multiple ways in which cultural strengths constitute a unique source of resilience for populations exposed to chronic adversity. As we have culturally adapted the parenting intervention for Latinx communities over the course of several years, we have followed the guidance from families to ensure the contextual and cultural relevance of adapted interventions.

In essence, our cultural adaptation protocols have been designed to facilitate a process characterized by mutual learning and shared expertise. Adopting such a stance is critical. On one hand, lack of adherence to PT principles that account for the efficacy of evidence-based interventions is very likely to result in deficient changes in parenting practices. Similarly, delivering PT components without contextual and cultural relevance carries the risk of becoming an experience of scientific imperialism, which is the main warning highlighted by Martín-Baró according to the premises of liberation psychology.

\section{Addressing Discrimination Inside and Outside the Therapy Room}

The US is experiencing unprecedented challenges associated with human rights violations of vulnerable Latina/o immigrants who are depicted as a burden to society, rather than pillars of US socioeconomic systems (McHugh, 2018). Children in these families are being impacted in unprecedented ways by continuous exposure to contextual stress and the traumatic effects of family separation (Pierce, Bolter, \& Selee, 2018).

In the face of this humanitarian crisis in a country that was founded on the premise of embracing immigrants fleeing persecution, family therapists must carefully reflect on their positionality as mental health providers. In our experience, we have confirmed the critical importance of overtly addressing discrimination in the parenting curricula. In addition, we have confirmed that exclusively limiting our scope of practice to promoting conversations focused on the adversity experienced by the families we serve can constitute an extension of the dynamics of oppression that they experience. Thus, we consider it essential to deliver mental health services to underserved populations within frameworks of advocacy, aimed at helping families access the resources they need to increase their coping skills to withstand the adversity they experience on a daily basis.

\section{CONCLUSION}

The proposed model illustrates an approach to intervention delivery characterized by social justice principles, evidence-based knowledge, family therapy theories, and cultural relevance. The model was also developed on the premise that parenting interventions can promote healing experiences for parents exposed to intense contextual oppression and 
adverse childhood backgrounds. Time and again, the families we serve have demonstrated to us how this type of models are ultimately grounded in reality thanks to their inspiring wisdom and courage. It is precisely these resilient parents who have continuously taught us that even in the midst of profound social injustice, lack of hope and despair can always be transformed into a promise for a liberating future.

\section{Acknowledgments}

Sincere thanks to Marion Forgatch, ISII Executive Director and Laura Rains, ISII Director of Implementation and Training. Their extraordinary support has been essential for the dissemination of Generation PMTO with underserved Latina/o populations. Deep gratitude to Karen S. and Richard S. Wampler, as well as James C. Anthony, for their inspiring mentoring and unwavering support.

I would like to express my deep gratitude to the courageous parents who have participated in our adapted interventions. This program of research would not be a reality without the strong commitment of our community partners, interventionists, project managers, and members of the research team. My deep gratitude to the many mentors who have supported this program of research, as well as to our committed Co-Investigators Cris Sullivan, Deborah Bybee, Guillermo Bernal, Brian Dates, Lisa Tams, and Melanie Domenech Rodríguez. The study was supported by funds from the National Institute on Mental Health R34MH087678 and National Institute on Drug Abuse (NIDA) K01DA036747 (to JRPC). In addition, NIDA grants K05DA015799 and T32DA021129 (to JCA). The content is solely the responsibility of the author and does not necessarily represent the official views of the National Institute of Mental Health, the National Institute on Drug Abuse, or the National Institutes of Health.

\section{REFERENCES}

Barrera M, Castro FG, Strycker LA, \& Toobert DJ (2013). Cultural adaptations of behavioral health interventions: A progress report. Journal of Consulting and Clinical Psychology, 81(2), 196-205. [PubMed: 22289132]

Bornstein MH (2012). Cultural approaches to parenting. Parenting: Science and Practice, 12, 212-221. 10.1080/15295192.2012.683359

Boszormenyi-Nagy I, \& Krasner BR (1986). Between give and take: A clinical guide to contextual therapy. Bristol, PA: Brunner/Mazel.

Burton LM, Bonilla-Silva E, Ray V, Buckelew R, \& Freeman EH (2010). Critical race theories, colorism, and the decade's research on families of color. Journal of Marriage and the Family, 72, 440-459. 10.1111/j.1741-3737.2010.00712.x

Cardoso JB, Scott JL, Faulkner M, \& Lane LB (2018). Parenting in the context of deportation. Journal of Marriage and the Family, 80, 301-316. 10.1111/jomf.12463.

Cassidy J (1999). The nature of the child's ties In Cassidy J \& Shaver PR (Eds.), Handbook of attachment: Theory, research, and clinical applications (pp. 3-20). New York: The Guilford Press.

Domenech Rodríguez MM, Baumann AA, \& Schwartz AL (2011). Cultural adaptation of an evidencebased intervention: From theory to practice in a Latino/a community context. American Journal of Community Psychology, 47, 170-186. 10.1007/s10464-010-9371-4 [PubMed: 21116707]

Forgatch MS, Bullock BM, \& Patterson GR (2004). From theory to practice: Increasing effective parenting through role-play: The Oregon model of parent management training (PMTO) In Steiner $\mathrm{H}$ (Ed.), Handbook of mental health interventions in children and adolescents: An integrated developmental approach (pp. 782-814). San Francisco, CA: Jossey-Bass.

Forgatch MS, \& Gewirtz AH (2018). The evolution of the Oregon model of parent management training: An intervention for antisocial behavior in children and adolescents In Weisz JR (Ed.), Evidence-based psychotherapies for children and adolescents (pp. 85-102). New York: Guilford Press.

Forgatch MS, Patterson GR, DeGarmo DS, \& Beldavs ZG (2009). Testing the Oregon delinquency model with 9-year follow-up of the Oregon divorce study. Development and Psychopathology, 21, 637-660. 10.1017/S0954579409000340 [PubMed: 19338702] 
Gerdes AC, Kapke TL, Lawton KE, Grace M, \& Hurtado GD (2015). Adapting parent training for Latino youth with ADHD: Development and pilot. Journal of Latina/o Psychology, 3, 71-87. 10.1037/lat0000037.

Hatzenbuehler ML, Prins SJ, Flake M, Philbin M, Frazer MS, Hagen D et al. (2017). Immigration policies and mental health morbidity among Latinos: A state-level analysis. Social Science and Medicine, 174, 169-178. 10.1016/j.socscimed.2016.11.040. [PubMed: 28043019]

Johnson BD, Berdahl LD, Horne M, Richter EA, \& Walters M (2014). A parenting competency model. Parenting: Science and Practice, 14, 92-120. 10.1080/15295192.2014.914361

Leijten P, Raaijmakers M, Wijngaards L, Matthys W, Menting A, \& Orobio de Castro B (2018). Understanding who benefits from parenting interventions for children's conduct problems: An integrative data analysis. Prevention Science, 19, 579-588. 10.1007/s11121-018-0864-y [PubMed: 29349546]

Martín Baró I (1977). Psicología, Ciencia, y Conciencia. San Salvador, El Salvador: UCA Editores.

McHugh M (2018). In the age of Trump: Populist backlash and progressive resistance create divergent sate immigrant integration contexts. Retrieved 1 11, 2019, from Migration Policy Institute website: https://www.migrationpolicy.org/research/age-trump-populist-backlash-and-progressiveresistance-create-divergent-state-immigrant

Michelson D, Davenport C, Dretzke J, Barlow J, \& Day C (2013). Do evidence-based interventions work when tested in the "real world?" A systematic review and meta-analysis of parent management training for the treatment of child disruptive behavior. Clinical Child and Family Psychology Review, 16, 18-34. 10.1007/s10567-013-0128-0 [PubMed: 23420407]

Park IJK, Du H, Wang L, Williams DR, \& Alegria M (2018). Racial/ethnic discrimination and mental health in Mexican-origin youths and their parents: Testing the "linked lives" hypothesis. Journal of Adolescent Health, 62, 480-487. 10.1016/j.jadohealth.2017.10.010. [PubMed: 29275862]

Parra-Cardona JR, Bybee D, Sullivan CM, Domenech Rodríguez MM, Dates B, Tams L et al. (2017). Examining the impact of differential cultural adaptation with Latina/o immigrants exposed to adapted parent training interventions. Journal of Consulting and Clinical Psychology, 85, 58-71. 10.1037/ccp0000160. [PubMed: 28045288]

Parra-Cardona JR, Domenech Rodríguez M, Forgatch MS, Sullivan C, Bybee D, Tams L et al. (2012). Culturally adapting an evidence-based parenting intervention for Latino immigrants: The need to integrate fidelity and cultural relevance. Family Process, 51, 56-72. 10.1111/j. 1545-5300.2012.01386.x. [PubMed: 22428711]

Parra-Cardona JR, López Zerón G, Domenech Rodríguez M, Escobar-Chew AR, Whitehead M, Sullivan C et al. (2016). A balancing act: Integrating evidence-based knowledge and cultural relevance in a program of prevention parenting research with Latino/a immigrants. Family Process, 55, 321-337. 10.1111/famp.12190. [PubMed: 26503301]

Parra-Cardona JR, Bybee D, Sullivan CM, Domenech Rodríguez MM, Dates B, Tams L, \& Bernal G (2017a). Examining the impact of differential cultural adaptation with Latina/o immigrants exposed to adapted parent training interventions. Journal of Consulting and Clinical Psychology, 85, 58-71. 10.1037/ccp0000160 [PubMed: 28045288]

Parra-Cardona JR, Lopez Zerón G, Villa M, Zamudio E, Escobar-Chew AR, \& Domenech Rodríguez M (2017b). Enhancing parenting practices with Latino/a parents: A community-based prevention model integrating evidence-based knowledge, cultural relevance, and advocacy. Clinical Social Work Journal, 45, 88-98. 10.1007/s10615-016-0589-y [PubMed: 28337047]

Parra-Cardona JR, López-Zerón G, Leija SG, Maas MK, Villa M, Zamudio E et al. (2018). Culturally adapted intervention for Mexican-origin parents of adolescents: The need to overtly address culture and discrimination in evidence-based practice. Family Process. Advance online publication. 10.1111/famp.12381

Peters MF (1985). Racial socialization of young Black children In McAdoo HP \& McAdoo JL (Eds.), Black children: Social, educational, and parental environments (pp. 159-173). Beverly Hills, CA: Sage.

Pierce S, Bolter J, \& Selee A (2018). U.S. immigration policy under Trump: Deep changes and lasting impacts. Retrieved on 1 11, 2019, from Migration Policy Institute website: https:// www.migrationpolicy.org/research/us-immigration-policy-trump-deep-changes-impacts 
Satir V, Banmen J, Gerber J, \& Gomori M (1991). The Satir model: Family therapy and beyond. Palo Alto, CA: Science and Behavior Books Inc.

Stein GL, \& Guzman LE (2015). Prevention and intervention research with Latino families: A translational approach. Family Process, 54, 280-292. 10.1111/famp.12143 [PubMed: 25735564]

Substance Abuse and Mental Health Services Administration (2015). Racial/ethnic differences in mental health service use among adults HHS Publication No. SMA-15-4906. Rockville, MD: Substance Abuse and Mental Health Services Administration.

Unger JB (2015). Preventing substance use and misuse among racial and ethnic minority adolescents: Why are we not addressing discrimination in prevention programs? Substance Use and Misuse, 50, 952-955. 10.3109/10826084.2015.1010903 [PubMed: 26361900]

Wong JY (2009). Understanding and utilizing parallel processes of social interaction for attachmentbased parenting interventions. Clinical Social Work Journal, 37, 163-174. 10.1007/ s10615-008-0155-3 


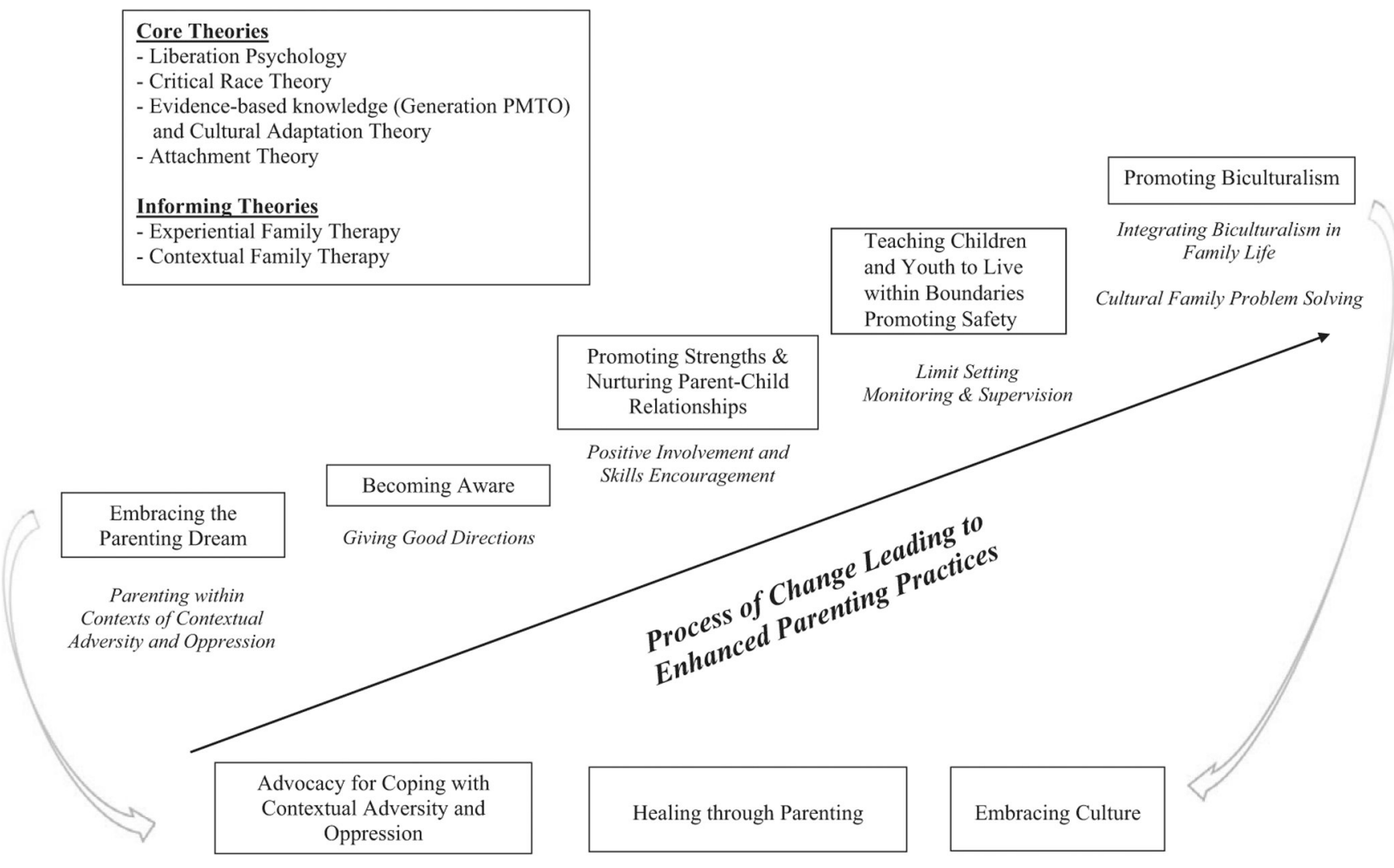

FIGURE 1.

Graphic Model of Intervention Delivery Approach and Process of Change. 